\title{
Opposite variation tendencies of serum CA724 levels in patients with colon and rectal carcinoma
}

\author{
ZHANMENG ZHU $^{1 *}$, ZHE CHEN $^{1 *}$, CHUNLIN CHEN $^{1}$, ZIYI YANG $^{2}$, WEIBO XUAN $^{2}$, \\ YAHUI HOU ${ }^{2}$, YUNFEI ZUO ${ }^{2}$ and SHUANGYI REN ${ }^{1}$ \\ ${ }^{1}$ Department of General Surgery, The Second Affiliated Hospital of Dalian Medical University, Dalian, Liaoning 116023; \\ ${ }^{2}$ Department of Clinical Biochemistry, Dalian Medical University, Dalian, Liaoning 116044, P.R. China
}

Received June 27, 2013; Accepted September 13, 2013

DOI: $10.3892 / \operatorname{mco} .2013 .208$

\begin{abstract}
The aim of this study was to investigate tumor biomarker carbohydrate antigen 724 (CA724) in the serum of patients with carcinomas of the colon and rectum at various clinical stages. Serum was collected from 51 patients with colon carcinoma (CC) and 49 patients with rectal carcinoma (RC). CA724 levels were then measured in the different groups according to site, TNM classification, gender, age and metastastic status of the patients. The statistical significance of the differences between the groups was calculated by non-parametric statistics (Mann-Whitney and Kruskall-Wallis tests). We observed a close association between the serum CA724 levels and tumor migration in colorectal carcinoma (CRC) and opposite variation tendencies of CA724 in the evolution of CC and RC. In conclusion, we identified a close association between the serum levels of CA724 and tumor migration in CRC. The opposite variation tendencies of CA724 in the different evolution groups of $\mathrm{CC}$ and $\mathrm{RC}$ may reflect the differences between these two types of cancer. The evaluation of serum CA724 may be of monitoring and and predictive value and may also assist in the development of treatment strategies for CRC patients.
\end{abstract}

Correspondence to: Professor Shuangyi Ren, Department of General Surgery, The Second Affiliated Hospital of Dalian Medical University, 467 Zhongshan Road, Dalian, Liaoning 116023, P.R. China

E-mail: rsydl@aliyun.com

Professor Yunfei Zuo, Department of Clinical Biochemistry, Dalian Medical University, 9 Lushunnan Road, Dalian, Liaoning 116044, P.R. China

E-mail: zyf04112002@aliyun.com

*Contributed equally

Key words: carbohydrate antigen 724 , colon carcinoma, rectal carcinoma, tumor markers

\section{Introduction}

Colorectal carcinoma (CRC) remains one of the most common malignancies worldwide and represents a global health problem (1). An increasing number of Asian countries, including China, Japan, South Korea and Singapore, have experienced a 2- to 4-fold increase in the incidence of CRC over the last few decades (2). The pathogenesis of CRC ordinarily occurs in a staged progression from normal colonic mucosa to adenoma and finally to carcinoma over a period of $\sim 7-10$ years (3-5). This sequenced progression over time provides an opportunity for early diagnosis and treatment.

It has previously been indicated that delayed diagnosis is the main reason for a poor prognosis (6). The traditional non-invasive and invasive methods of screening modalities include fecal occult blood testing, fecal immunochemical test, double-contrast barium enema, flexible sigmoidoscopy and colonoscopy (7-9). Although some of these screening modalities have been demonstrated to reduce the rates of malignancy or mortality, there remains the issue of reducing cancer-related mortality by removing premalignant adenomas and early localized cancer prior to the onset of more advanced stages. Therefore, an effective approach to early screening, diagnosis and follow-up monitoring of CRC is required.

Over the last few years, extensive investigations have focused on serum tumor markers (STMs). In patients with CRC, single STMs exhibit low sensitivity and specificity, whereas the simultaneous measurement of several STMs may increase their diagnostic accuracy (10). It was demonstrated that the combined use of carcionembryonic antigen (CEA) and carbohydrate antigen (CA) 19-9 was effective in the screening and diagnosis of CRC (11). CA724 was previously identified as a type of STM specific for gastric cancer (12) and the correlation between CA724 and CRC has been attracting increasing attention (13).

Furthermore, due to the numerous characteristics shared by colon carcinoma (CC) and rectal carcinoma (RC), the two are discussed as a single entity. Whether $\mathrm{CC}$ and $\mathrm{RC}$ should be considered as a single or two distinct entities remains controversial (14). The aim of the present study was to investigate serum CA724 levels in patients with different clinical stages of $\mathrm{CC}$ and $\mathrm{RC}$ and analyze the correlation between serum CA724 levels and the clinical stages of CRC. 


\section{Patients and methods}

Patients. In this study, a total of 100 patients (63 male and 37 female) with histologically confirmed CRC were investigated. The patient sample was comprised of 51 patients with CC (32 male and 19 female) and 49 patients with RC ( 31 male and 18 female). The CC and RC patients were classified into four stages according to the 2003 TNM classification. Stages I and II were considered as early-stage, whereas stages III and IV were considered as advanced-stage disease. The patients in the advanced-stage group had either lymph node (stage III) or distant metastases (stage IV). Patient information, such as gender, age and pathological TNM staging, is summarized in Table I. The ethics committee for the Second Affiliated Hospital of Dalian Medical University provided approval for this study (Dalian, China)

Serum collection and CA724 assay. The values of CA724 were measured prior to the patients receiving radiation treatment or chemotherapy. Blood samples were collected, separated by centrifugation and the serum samples were stored at $-20^{\circ} \mathrm{C}$ until assays were performed. The CA724 kit was provided by Diagnostic Products Corporation (DPC, Tianjin, China). The serum CA724 levels were determined with an immunoradiometric gamma counter (DPC-GAMMA-C12; DPC). The patient details were furnished by the First and Second Hospitals affiliated to Dalian Medical University, between 2010 and 2012.

Statistical analysis. The CA724 levels were assessed in different groups according to different site, TNM classification, gender, age and metastatic status of the patients. Since the serum CA724 levels in each group did not follow a normal distribution, the statistical significance of the differences between the groups was calculated by non-parametric statistics (Mann-Whitney and Kruskall-Wallis tests). The centralized tendency of each group was described as means plus standard error of the mean (SEM). All statistical tests were performed using SPSS software, version 11.5 (SPSS Inc., Chicago, IL, USA). P<0.05 was considered to indicate a statistically significant difference.

\section{Results}

Differences among CRC patients in different groups. The mean serum CA724 values \pm SEM were $6.808 \pm 1.462 \mathrm{U} / \mathrm{ml}$ in $\mathrm{CC}$ patients and $5.524 \pm 1.151 \mathrm{U} / \mathrm{ml}$ in RC patients, with no statistically significant difference. Therefore, we first analyzed the two types of cancer as a single entity. The different CA724 levels among CRC patients in different groups are presented in Table II. The differences in the CA724 values between CRC clinical stages were compared (Fig. 1). Further investigations indicated that the mean serum CA724 concentrations \pm SEM in patients with early- and advanced-stage disease were $5.414 \pm 1.317$ and $6.689 \pm 1.281 \mathrm{U} / \mathrm{ml}$, respectively. The differences between the two groups were not statistically significant. However, the differences between the CRC patient group with and that without distant metastasis was statistically significant $(\mathrm{P}=0.043)$.

To determine whether gender and age affected the results in CRC patients mentioned above, the differences in the serum
Table I. Clinicopathological characteristics of 100 CRC patients.

\begin{tabular}{lcc}
\hline Variables & No. of CC & No. of RC \\
\hline Gender & 32 & 31 \\
Male & 19 & 18 \\
Female & & \\
Age (years) & 9 & 11 \\
$\leq 50$ & 42 & 38 \\
$>50$ & & \\
Lymph node metastasis & 18 & 22 \\
No & 33 & 27 \\
Yes & & \\
Distant metastasis & 35 & 39 \\
No & 16 & 10 \\
Yes & & \\
Stage & 6 & 14 \\
I & 12 & 8 \\
II & 17 & 10 \\
III & 16 & \\
IV & & \\
\hline
\end{tabular}

$\mathrm{CC}$, colon carcinoma; $\mathrm{RC}$, rectal carcinoma; $\mathrm{CRC}$, colorectal carcinoma.

Table II. Differences in CA724 levels among patients with colorectal carcinoma in different groups.

\begin{tabular}{lcc}
\hline Variables & Pt no. (CA724 levels $)$ & P-value \\
\hline Site & & NS \\
Colon & $51(6.808 \pm 1.462)$ & \\
Rectum & $49(5.524 \pm 1.151)$ & \\
Lymph node metastasis & & NS \\
No & $40(5.414 \pm 1.317)$ & \\
Yes & $60(6.689 \pm 1.281)$ & \\
Distant metastasis & & 0.043 \\
No & $74(4.835 \pm 0.791)$ & \\
Yes & $26(10.005 \pm 2.677)$ & \\
Stage & & NS \\
I & $20(7.464 \pm 2.156)$ & \\
II & $20(3.364 \pm 1.426)$ & \\
III & $34(4.153 \pm 0.760)$ & \\
IV & $26(10.005 \pm 2.677)$ & \\
Gender & & NS \\
Male & $63(5.771 \pm 1.039)$ & \\
Female & $37(7.148 \pm 1.798)$ & \\
Age & & NS \\
$30-50$ & $20(5.026 \pm 1.560)$ & \\
$>50$ & $80(6.467 \pm 1.096)$ & \\
\hline
\end{tabular}

${ }^{a}$ Mean \pm standard error of the mean, U/ml. Pt, patient; CA, carbohydrate antigen; NS, not significant. 

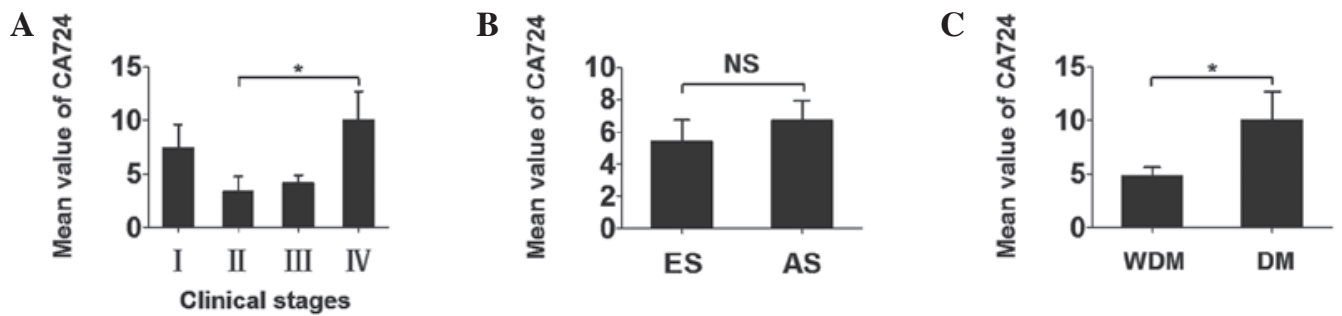

Figure 1. Different mean carbohydrate antigen (CA) 724 values among colorectal carcinoma (CRC) patients in different groups. (A) The graph shows the variation trend of CA724 levels among the four different clinical stages. Only the differences between stages II and IV were statistically significant ( $\mathrm{P}=0.025)$. (B) There were no statistically significant differences between patients with early-stage (ES) and advanced-stage (AS) disease. (C) There were statistically significant differences between patients without distant metastasis (WDM) and those with distant metastasis $(\mathrm{DM})\left({ }^{*} \mathrm{P}=0.043\right)$. The results are presented as means + standard error of the mean.
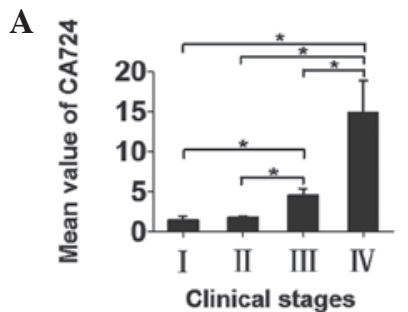

B

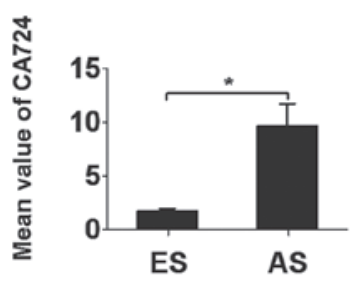

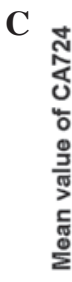

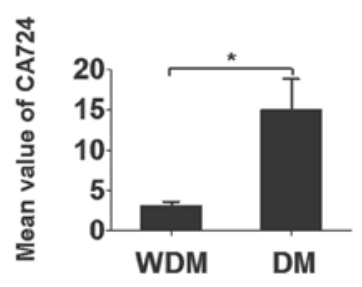

Figure 2. Different mean carbohydrate antigen (CA) 724 values among colon carcinoma patients in different groups. (A) The graph shows an ascending variation trend of CA724 values with increasing clinical stage. Multiple comparisons among clinical stages revealed statistically significant differences $(* \mathrm{P}<0.05)$, except between stages I and II. (B) The CA724 values in early-stage (ES) were higher compared to those in advanced-stage (AS) disease, with a statistically significant difference ( $\left.{ }^{*} \mathrm{P}<0.001\right)$. (C) A similar phenomenon was observed between patients with distant metastasis (DM) and those without distant metastasis (WDM). This difference was also considered to be statistically significant $\left({ }^{*} \mathrm{P}<0.001\right)$. The results are presented as means + standard error of the mean.
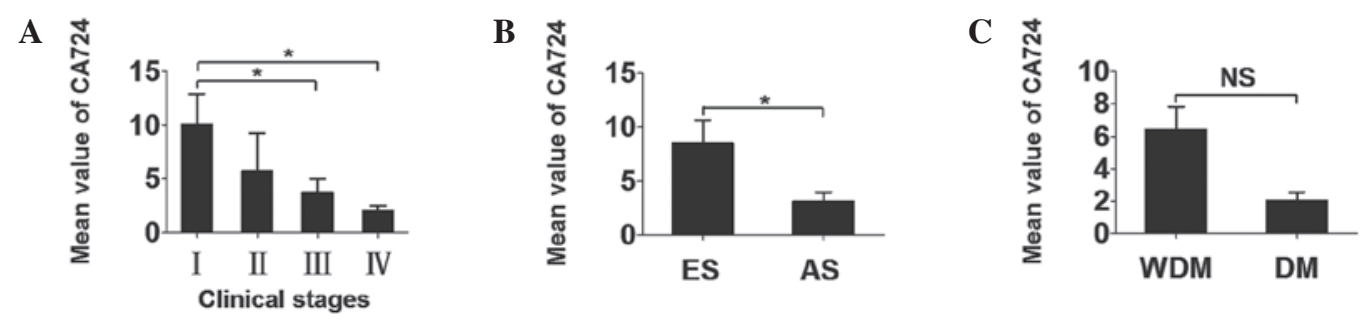

Figure 3. Different mean carbohydrate antigen (CA) 724 values among rectal carcinoma patients in different groups. (A) The graph shows a descending variation trend of CA724 values with increasing clinical stage. Multiple comparisons among clinical stages indicate that the differences between stages I and III ("P=0.003) and those between stages I and IV (" $\mathrm{P}=0.009)$ reached a statistical significance. (B) The level of CA724 values in early-stage (ES) were lower compared to those in advanced-stage (AS) disease, with a statistically significant difference ( $\mathrm{P}=0.011$ ). (C) A similar phenomenon was observed between patients with distant metastasis (DM) and those without distant metastasis (WDM). However, the difference was not statistically significant. The results are presented as means + standard error of the mean.

CA724 values between genders was also assessed. Table III shows that there were no significant differences between male and female CRC patients. Furthermore, there were no significant differences between CRC patients aged 30-50 years and those aged $>50$ years. Therefore, the variables of gender and age were eliminated (Table II). Since there were distinct differences among CRC patients, in order to interpret the differences described above when assessing the two groups as a single entity, we proceeded to analyze CC and RC separately.

Ascending gradient among CC patients. The different CA724 levels among CC patients in different groups are presented in Table III. There was an ascending gradient of serum CA724 values with increasing clinical stage, except for the difference between stages I and II (Fig. 2). The mean CA724 values \pm SEM for each stage were as follows: stage I, $1.437 \pm 0.535 \mathrm{U} / \mathrm{ml}$; stage II, $1.777 \pm 0.249 \mathrm{U} / \mathrm{ml}$; stage III, $4.559 \pm 0.855 \mathrm{U} / \mathrm{ml}$; and stage IV, $14.985 \pm 3.875 \mathrm{U} / \mathrm{ml}$. The differences in the CA724 values between early- and advanced-stage disease were statistically significant $(\mathrm{P}<0.001)$, as were those between patients with and those without distant metastasis $(\mathrm{P}<0.001)$ (Fig. 2).

Descending gradient among RC patients. The different CA724 levels among CC patients in different groups are presented in Table IV. The mean serum CA724 values \pm SEM for each stage were as follows: stage I, 10.046 $\pm 2.819 \mathrm{U} / \mathrm{ml}$; stage II, $5.745 \pm 3.506 \mathrm{U} / \mathrm{ml}$; stage III, $3.748 \pm 1.277 \mathrm{U} / \mathrm{ml}$; and stage IV, $2.036 \pm 0.491 \mathrm{U} / \mathrm{ml}$, these results are based on the data provided in Table V. Therefore, a descending gradient was identified among RC patients when each clinical stage was separately analyzed (Fig. 3). Similarly, analysis with SPSS software, version 11.5, revealed a significant correlation between early- and advanced-stage disease $(\mathrm{P}=0.010)$. However, the differences in the CA724 values between patients with and those without distant metastasis did not reach a statistical significance (Fig. 3). 
Table III. Differences in CA724 levels among CC patients.

\begin{tabular}{lcc}
\hline Variables & Pt no. (CA724 levels $\left.{ }^{\mathrm{a}}\right)$ & P-value \\
\hline Lymph node metastasis & $18(1.663 \pm 0.237)$ & $<0.001$ \\
No & $33(9.614 \pm 2.109)$ & \\
Yes & & $<0.001$ \\
Distant metastasis & $35(3.143 \pm 0.493)$ & \\
No & $16(14.985 \pm 3.875)$ & \\
Yes & & $<0.001$ \\
Stage & $6(1.437 \pm 0.535)$ & \\
I & $12(1.777 \pm 0.249)$ & \\
II & $17(4.559 \pm 0.855)$ & \\
III & $16(14.985 \pm 3.875)$ & \\
IV & & NS \\
Gender & $32(6.451 \pm 1.508)$ & \\
Male & $19(7.409 \pm 3.051)$ & \\
Female & & \\
Age (years $)$ & $9(4.664 \pm 1.160)$ & \\
$30-50$ & $42(7.267 \pm 1.755)$ & \\
$>50$ & & \\
\hline
\end{tabular}

${ }^{\mathrm{a}}$ Mean $\pm \mathrm{SEM}, \mathrm{U} / \mathrm{ml}$. Pt, patient; CC, colon carcinoma; CA, carbohydrate antigen; NS, not significant.

Table IV. Differences in CA724 levels among RC patients.

\begin{tabular}{lcc}
\hline Variables & Pt no. $\left(\right.$ CA724 levels $\left.{ }^{\mathrm{a}}\right)$ & P-value \\
\hline Lymph node metastasis & $22(8.482 \pm 2.196)$ & 0.011 \\
No & $27(3.114 \pm 0.830)$ & \\
Yes & & NS \\
Distant metastasis & $39(6.419 \pm 1.396)$ & \\
No & $10(2.036 \pm 0.491)$ & \\
Yes & & 0.022 \\
Stage & $14(10.046 \pm 2.819)$ & \\
I & $8(5.745 \pm 3.506)$ & \\
II & $17(3.748 \pm 1.277)$ & \\
III & $10(2.036 \pm 0.491)$ & \\
IV & & NS \\
Gender & $31(5.069 \pm 1.442)$ & \\
Male & $18(6.308 \pm 1.905)$ & \\
Female & & NS \\
Age (years & $11(5.322 \pm 2.739)$ & \\
$30-50$ & $38(5.583 \pm 1.261)$ & \\
$>50$ & &
\end{tabular}

${ }^{\mathrm{a}}$ Mean \pm SEM, U/ml. Pt, patient; RC, rectal carcinoma; CA, carbohydrate antigen; NS, not significant.

\section{Discussion}

This comprehensive integrative analysis of $100 \mathrm{CRC}$ patients indicated that serum CA724 levels may be of predictive value in CRC, particularly in the analysis of clinical stage. As regards CA724, recent studies have mainly focused on the sensitivity and specificity of its diagnostic and early detection value for recurrences. The combinations of CA724 with other STMs were considered to be satisfactory $(10,13,15-17)$. In our study, we focused on the differences in the CA724 levels among patients with different disease stages.

The differences in serum CA724 values between patients with early- and advanced-stage disease suggest that CA724 may be associated with the metastasis of $\mathrm{CC}$ and RC. A similar association was observed between CEA levels and CRC metastasis $(18,19)$. When comparing and analysing CC and RC, we may surmise that the differences in the serum levels of CA724 represent a sign of distant metastasis in CC. However, we did not observe a statistically significant difference between RC patients with and those without distant metastasis. We then compared the clinical stages of $\mathrm{CC}$ and RC. Although we were unable to verify a statistically significant difference for each stage transition, there was a distinct variation tendency among the stages. Therefore, we consider that monitoring serum CA724 levels may be indicative of clinical stage, particularly in patients for whom the determination of the pathological stage is difficult. The CA724 value may reflect advanced stage, progression and metastasis of CRC. Due to the widely variable prognosis of CRC, a previous study attempted to identify a parameter useful in the selection of patients who may be candidates for more tailored treatment (20). Whether CA724 is such a parameter requires further verification; however, our results suggest that it has potential as a tumor marker. Considering the association between CA724 and CRC, we may surmise the presence of similar associations between other STMs and carcinomas.

Our study demonstrated the presence of two opposite trends in the levels of CA724 according to the progression of the clinical CRC stage. As was mentioned previously, there was an ascending gradient among CC patients and a descending gradient among RC patients. These two opposite trends possibly reflect biological, advancing and metastatic differences between CC and RC. Due to the similarities in morphology and configuration and the fact that one is considered to be the continuation of the other, $\mathrm{CC}$ and $\mathrm{RC}$ are often considered as a single disease entity. However, an increasing number of studies refer to the differences between these two types of cancer. Firstly, the colon embryologically originates from the midgut and hindgut, whereas the rectum originates from the cloaca. Furthermore, relevant studies have demonstrated that there were differences regarding blood supply, biological function, histochemical reactions and the level of mRNA expression (21-26). Those studies indicated that the normal colon and rectum are different. A previous study also reported that the prognosis of $\mathrm{CC}$ is better compared to that of RC and that RC exhibits a higher expression of CEA15. Additionally, a gene-level analysis demonstrated that methylation and mutations were more common in the right colon (27). All those results suggested that there are biological differences between $\mathrm{CC}$ and RC. The exact mechanism underlying the descending gradient in the CA724 levels among RC patients has not been elucidated.

Although this study included a small number of patients, the results regarding the association between CA724 levels 
Table V. Clinicopathological characteristics of 100 patients with colorectal carcinoma.

\begin{tabular}{|c|c|c|c|c|c|c|}
\hline No. & Pathological diagnosis & Site & Pathological TNM staging & Lesion & Age/gender & CA724 \\
\hline 1 & Adenocarcinoma & Colon & T2N0M0 & Primary tumor & 61/M & 4.05 \\
\hline 2 & Adenocarcinoma & Colon & T2NOM0 & Primary tumor & 77/M & 1.19 \\
\hline 3 & Adenocarcinoma & Colon & T2NOM0 & Primary tumor & $61 / \mathrm{M}$ & 1.13 \\
\hline 4 & Adenocarcinoma & Colon & T1N0M0 & Primary tumor & $63 / \mathrm{F}$ & 1.09 \\
\hline 5 & Adenocarcinoma & Colon & T2N0M0 & Primary tumor & 69/M & 0.59 \\
\hline 6 & Adenocarcinoma & Colon & T2N0M0 & Primary tumor & $58 / \mathrm{F}$ & 0.57 \\
\hline 7 & Adenocarcinoma & Colon & T4N0M0 & Primary tumor & $65 / F$ & 3.44 \\
\hline 8 & Adenocarcinoma & Colon & T4N0M0 & Primary tumor & $61 / F$ & 3.04 \\
\hline 9 & Adenocarcinoma & Colon & T4N0M0 & Primary tumor & $56 / \mathrm{F}$ & 2.78 \\
\hline 10 & Adenocarcinoma & Colon & T3N0M0 & Primary tumor & $75 / F$ & 1.81 \\
\hline 11 & Adenocarcinoma & Colon & T4N0M0 & Primary tumor & 64/M & 1.71 \\
\hline 12 & Adenocarcinoma & Colon & T3N0M0 & Primary tumor & 48/M & 1.67 \\
\hline 13 & Adenocarcinoma & Colon & T3N0M0 & Primary tumor & 33/M & 1.49 \\
\hline 14 & Adenocarcinoma & Colon & T4N0M0 & Primary tumor & $51 / \mathrm{F}$ & 1.38 \\
\hline 15 & Adenocarcinoma & Colon & T3N0M0 & Primary tumor & $55 / \mathrm{M}$ & 1.24 \\
\hline 16 & Adenocarcinoma & Colon & T4N0M0 & Primary tumor & 67/M & 1.10 \\
\hline 17 & Adenocarcinoma & Colon & T3N0M0 & Primary tumor & 69/M & 0.85 \\
\hline 18 & Adenocarcinoma & Colon & T4N0M0 & Primary tumor & $48 / \mathrm{F}$ & 0.81 \\
\hline 19 & Adenocarcinoma & Colon & T4N1M0 & Primary tumor & $65 / \mathrm{M}$ & 11.90 \\
\hline 20 & Adenocarcinoma & Colon & T3N1M0 & Primary tumor & 64/M & 10.22 \\
\hline 21 & Adenocarcinoma & Colon & T3N1M0 & Primary tumor & $45 / \mathrm{M}$ & 8.92 \\
\hline 22 & Adenocarcinoma & Colon & T4N1M0 & Primary tumor & $52 / \mathrm{F}$ & 8.44 \\
\hline 23 & Adenocarcinoma & Colon & T3N1M0 & Primary tumor & $60 / \mathrm{F}$ & 6.44 \\
\hline 24 & Adenocarcinoma & Colon & T4N1M0 & Primary tumor & $45 / F$ & 6.31 \\
\hline 25 & Adenocarcinoma & Colon & T3N1M0 & Primary tumor & $51 / \mathrm{M}$ & 4.78 \\
\hline 26 & Adenocarcinoma & Colon & T3N2M0 & Primary tumor & 61/M & 3.86 \\
\hline 27 & Adenocarcinoma & Colon & T3N2M0 & Primary tumor & $62 / F$ & 3.38 \\
\hline 28 & Adenocarcinoma & Colon & T3N1M0 & Primary tumor & 35/M & 2.90 \\
\hline 29 & Adenocarcinoma & Colon & T3N2M0 & Primary tumor & $55 / \mathrm{M}$ & 2.05 \\
\hline 30 & Adenocarcinoma & Colon & T3N2M0 & Primary tumor & $54 / \mathrm{F}$ & 1.82 \\
\hline 31 & Adenocarcinoma & Colon & T3N1M0 & Primary tumor & $70 / \mathrm{F}$ & 1.74 \\
\hline 32 & Adenocarcinoma & Colon & T3N1M0 & Primary tumor & 68/M & 1.67 \\
\hline 33 & Adenocarcinoma & Colon & T3N1M0 & Primary tumor & 67/M & 1.09 \\
\hline 34 & Adenocarcinoma & Colon & T3N1M0 & Primary tumor & $66 / \mathrm{M}$ & 1.03 \\
\hline 35 & Adenocarcinoma & Colon & T4N1M0 & Primary tumor & $67 / F$ & 0.95 \\
\hline 36 & Adenocarcinoma & Colon & T2N0M1 & Primary tumor & $66 / F$ & 57.79 \\
\hline 37 & Adenocarcinoma & Colon & T3N0M1 & Primary tumor & $81 / \mathrm{M}$ & 43.30 \\
\hline 38 & Adenocarcinoma & Colon & T2N0M1 & Primary tumor & $70 / \mathrm{F}$ & 19.99 \\
\hline 39 & Adenocarcinoma & Colon & T4N2M1 & Primary tumor & 61/M & 19.90 \\
\hline 40 & Adenocarcinoma & Colon & TxNxM1 & Primary tumor & $64 / F$ & 16.56 \\
\hline 41 & Adenocarcinoma & Colon & T4N2M1 & Primary tumor & $56 / \mathrm{M}$ & 16.17 \\
\hline 42 & Adenocarcinoma & Colon & TxNxM1 & Primary tumor & $59 / \mathrm{M}$ & 15.15 \\
\hline 43 & Adenocarcinoma & Colon & T4N1M1 & Primary tumor & $80 / \mathrm{M}$ & 12.23 \\
\hline 44 & Adenocarcinoma & Colon & T3N2M1 & Primary tumor & 37/M & 10.76 \\
\hline 45 & Adenocarcinoma & Colon & T4N2M1 & Primary tumor & 75/M & 7.37 \\
\hline 46 & Adenocarcinoma & Colon & TxNxM1 & Primary tumor & 74/M & 7.03 \\
\hline 47 & Adenocarcinoma & Colon & T4N1M1 & Primary tumor & $42 / \mathrm{M}$ & 5.54 \\
\hline 48 & Adenocarcinoma & Colon & T4N0M1 & Primary tumor & $42 / \mathrm{M}$ & 3.58 \\
\hline 49 & Adenocarcinoma & Colon & T4N0M1 & Primary tumor & $74 / \mathrm{F}$ & 2.43 \\
\hline 50 & Adenocarcinoma & Colon & TxNxM1 & Primary tumor & $36 / \mathrm{M}$ & 1.32 \\
\hline 51 & Adenocarcinoma & Colon & T3N0M1 & Primary tumor & $61 / \mathrm{M}$ & 0.64 \\
\hline
\end{tabular}


Table V. Continued.

\begin{tabular}{|c|c|c|c|c|c|c|}
\hline No. & Pathological diagnosis & Site & Pathological TNM staging & Lesion & Age/gender & CA724 \\
\hline 52 & Adenocarcinoma & Rectum & T2N0M0 & Primary tumor & $66 / \mathrm{M}$ & 29.60 \\
\hline 53 & Adenocarcinoma & Rectum & T2NOM0 & Primary tumor & $72 / \mathrm{M}$ & 26.03 \\
\hline 54 & Adenocarcinoma & Rectum & T2N0M0 & Primary tumor & $79 / \mathrm{M}$ & 24.86 \\
\hline 55 & Adenocarcinoma & Rectum & T2NOM0 & Primary tumor & $56 / \mathrm{F}$ & 18.30 \\
\hline 56 & Adenocarcinoma & Rectum & T2NOM0 & Primary tumor & $55 / \mathrm{M}$ & 16.02 \\
\hline 57 & Adenocarcinoma & Rectum & T2NOM0 & Primary tumor & $67 / \mathrm{F}$ & 6.48 \\
\hline 58 & Adenocarcinoma & Rectum & T2NOM0 & Primary tumor & $73 / \mathrm{F}$ & 3.93 \\
\hline 59 & Adenocarcinoma & Rectum & T2NOM0 & Primary tumor & $65 / \mathrm{M}$ & 3.91 \\
\hline 60 & Adenocarcinoma & Rectum & T2NOM0 & Primary tumor & $57 / \mathrm{M}$ & 2.35 \\
\hline 61 & Adenocarcinoma & Rectum & T2N0M0 & Primary tumor & 78/M & 2.14 \\
\hline 62 & Adenocarcinoma & Rectum & T2N0M0 & Primary tumor & $62 / \mathrm{F}$ & 2.07 \\
\hline 63 & Adenocarcinoma & Rectum & T2N0M0 & Primary tumor & $59 / \mathrm{M}$ & 1.95 \\
\hline 64 & Adenocarcinoma & Rectum & T2N0M0 & Primary tumor & $79 / \mathrm{F}$ & 1.88 \\
\hline 65 & Adenocarcinoma & Rectum & T2N0M0 & Primary tumor & $72 / \mathrm{F}$ & 1.13 \\
\hline 66 & Adenocarcinoma & Rectum & T3N0M0 & Primary tumor & $55 / \mathrm{F}$ & 29.85 \\
\hline 67 & Adenocarcinoma & Rectum & T3N0M0 & Primary tumor & $67 / \mathrm{F}$ & 5.92 \\
\hline 68 & Adenocarcinoma & Rectum & T4N0M0 & Primary tumor & $54 / \mathrm{M}$ & 4.27 \\
\hline 69 & Adenocarcinoma & Rectum & T3N0M0 & Primary tumor & $55 / \mathrm{F}$ & 2.06 \\
\hline 70 & Adenocarcinoma & Rectum & T3N0M0 & Primary tumor & $67 / \mathrm{M}$ & 1.40 \\
\hline 71 & Adenocarcinoma & Rectum & T3N0M0 & Primary tumor & $66 / F$ & 0.95 \\
\hline 72 & Adenocarcinoma & Rectum & T3N0M0 & Primary tumor & $67 / F$ & 0.83 \\
\hline 73 & Adenocarcinoma & Rectum & T4N0M0 & Primary tumor & $73 / \mathrm{M}$ & 0.68 \\
\hline 74 & Adenocarcinoma & Rectum & T4N2M0 & Primary tumor & $38 / \mathrm{F}$ & 15.27 \\
\hline 75 & Adenocarcinoma & Rectum & T3N1M0 & Primary tumor & $79 / \mathrm{F}$ & 14.91 \\
\hline 76 & Adenocarcinoma & Rectum & T3N1M0 & Primary tumor & $68 / \mathrm{M}$ & 13.70 \\
\hline 77 & Adenocarcinoma & Rectum & T4N2M0 & Primary tumor & $48 / \mathrm{M}$ & 3.14 \\
\hline 78 & Adenocarcinoma & Rectum & T3N1M0 & Primary tumor & 72/M & 3.13 \\
\hline 79 & Adenocarcinoma & Rectum & T4N2M0 & Primary tumor & 73/M & 2.93 \\
\hline 80 & Adenocarcinoma & Rectum & T4N1M0 & Primary tumor & $57 / \mathrm{M}$ & 1.48 \\
\hline 81 & Adenocarcinoma & Rectum & T3N1M0 & Primary tumor & $55 / \mathrm{M}$ & 1.14 \\
\hline 82 & Adenocarcinoma & Rectum & T2N1M0 & Primary tumor & 71/M & 1.13 \\
\hline 83 & Adenocarcinoma & Rectum & T3N1M0 & Primary tumor & $54 / \mathrm{M}$ & 1.05 \\
\hline 84 & Adenocarcinoma & Rectum & T3N1M0 & Primary tumor & $47 / \mathrm{F}$ & 1.00 \\
\hline 85 & Adenocarcinoma & Rectum & T3N1M0 & Primary tumor & 48/M & 0.96 \\
\hline 86 & Adenocarcinoma & Rectum & T4N1M0 & Primary tumor & 69/M & 0.91 \\
\hline 87 & Adenocarcinoma & Rectum & T4NxM0 & Primary tumor & $76 / \mathrm{M}$ & 0.86 \\
\hline 88 & Adenocarcinoma & Rectum & T4N1M0 & Primary tumor & $43 / \mathrm{M}$ & 0.80 \\
\hline 89 & Adenocarcinoma & Rectum & T3N2M0 & Primary tumor & $44 / \mathrm{M}$ & 0.67 \\
\hline 90 & Adenocarcinoma & Rectum & T3N2M0 & Primary tumor & 72/M & 0.63 \\
\hline 91 & Adenocarcinoma & Rectum & TxNxM1 & Primary tumor & $74 / \mathrm{F}$ & 5.80 \\
\hline 92 & Adenocarcinoma & Rectum & TxN1M1 & Primary tumor & $54 / \mathrm{M}$ & 2.34 \\
\hline 93 & Adenocarcinoma & Rectum & T4N1M1 & Primary tumor & $67 / F$ & 0.95 \\
\hline 94 & Adenocarcinoma & Rectum & T3N1M1 & Primary tumor & $62 / \mathrm{M}$ & 3.33 \\
\hline 95 & Adenocarcinoma & Rectum & T4NxM1 & Primary tumor & $43 / \mathrm{F}$ & 1.59 \\
\hline 96 & Adenocarcinoma & Rectum & T4NxM1 & Primary tumor & 61/M & 2.26 \\
\hline 97 & Adenocarcinoma & Rectum & T4N1M1 & Primary tumor & 67/M & 1.32 \\
\hline 98 & Adenocarcinoma & Rectum & TxNxM1 & Primary tumor & 39/M & 1.10 \\
\hline 99 & Adenocarcinoma & Rectum & T4NxM1 & Primary tumor & $62 / \mathrm{M}$ & 1.04 \\
\hline 100 & Adenocarcinoma & Rectum & T3N2M4 & Primary tumor & $53 / \mathrm{F}$ & 0.63 \\
\hline
\end{tabular}

Age is presented in years and CA724 values in U/ml. CA, carbohydrate antigen; M, male; F, female. 
and CRC patients were considered to be statistically significant. Due to the small number of stage I CC patients $(n=6)$, further investigations are required to confirm these findings. Furthermore, the exact nature of the association and the underlying pathophysiological mechanisms of the opposite trends of serum CA724 in CC and $\mathrm{RC}$ require further investigation by future large prospective studies.

In conclusion, we demonstrated a close correlation between serum CA724 levels and tumor migration in CRC. The opposite variation tendencies of CA724 levels in the different evolution groups of $\mathrm{CC}$ and $\mathrm{RC}$ may reflect the differences between these two types of cancer. The evaluation of serum CA724 levels, particularly elevation in patients with CC, may be of monitoring and predictive value and may also assist in the development of treatment strategies for CRC patients.

\section{Acknowledgements}

This study was supported by grants from NSFC (31270867), the State Key Development Program of Basic Research of China (2012CB822103) and the Department of Science and Technology for Liaoning Province (2012225020).

\section{References}

1. Jemal A, Siegel R, Ward E, et al: Cancer statistics, 2009. CA Cancer J Clin 59: 225-249, 2009.

2. Sung JJ, Lau JY, Goh KL, et al: Increasing incidence of colorectal cancer in Asia: implications for screening. Lancet Oncol 6: 871-876, 2005.

3. Hofstad B and Vatn M: Growth rate of colon polyps and cancer. Gastrointest Endosc Clin N Am 7: 345-363, 1997.

4. Winawer SJ, Fletcher RH, Miller L, et al: Colorectal cancer screening: clinical guidelines and rationale. Gastroenterology 112: 594-642, 1997.

5. Wong JJ, Hawkins NJ and Ward RL: Colorectal cancer: a model for epigenetic tumorigenesis. Gut 56: 140-148, 2007.

6. Diallo Owono FK, Nguema Mve R, Ibaba J, Mihindou C and Ondo N'dong F: Epidemiological and diagnostic features of colorectal cancer in Libreville, Gabon. Med Trop (Mars) 71 605-607, 2011 (In French).

7. U.S. Preventive Services Task Force: Screening for colorectal cancer: U.S. Preventive Services Task Force recommendation statement. Ann Intern Med 149: 627-637, 2008.

8. Levin B, Lieberman DA, McFarland B, et al; American Cancer Society Colorectal Cancer Advisory Group; US Multi-Society Task Force; American College of Radiology Colon Cancer Committee: Screening and surveillance for the early detection of colorectal cancer and adenomatous polyps, 2008: a joint guideline from the American Cancer Society, the US Multi-Society Task Force on Colorectal Cancer, and the American College of Radiology. CA Cancer J Clin 58: 130-160, 2008.

9. Dominic OG, McGarrity T, Dignan M and Lengerich EJ: American College of Gastroenterology Guidelines for Colorectal Cancer Screening 2008. Am J Gastroenterol 104: 2626-2627; author reply 2628-2629, 2009.
10. Lumachi F, Marino F, Orlando R, Chiara GB and Basso SM: Simultaneous multianalyte immunoassay measurement of five serum tumor markers in the detection of colorectal cancer. Anticancer Res 32: 985-988, 2012.

11. Wang JY, Lu CY, Chu KS, et al: Prognostic significance of pre- and postoperative serum carcinoembryonic antigen levels in patients with colorectal cancer. Eur Surg Res 39: 245-250, 2007.

12. Chen XZ, Zhang WK, Yang K, et al: Correlation between serum CA724 and gastric cancer: multiple analyses based on Chinese population. Mol Biol Rep 39: 9031-9039, 2012.

13. Nicolini A, Ferrari P, Duffy MJ, et al: Intensive risk-adjusted follow-up with the CEA, TPA, CA19.9, and CA72.4 tumor marker panel and abdominal ultrasonography to diagnose operable colorectal cancer recurrences: effect on survival. Arch Surg 145: 1177-1183, 2010.

14. Li M, Li JY, Zhao AL and Gu J: Colorectal cancer or colon and rectal cancer? Clinicopathological comparison between colonic and rectal carcinomas. Oncology 73: 52-57, 2007.

15. Filella X, Molina R, Mengual PJ, et al: Significance of CA72.4 in patients with colorectal cancer. Comparison with CEA and CA19.9. J Nucl Biol Med 35: 158-161, 1991.

16. Yu JK, Yang MQ, Jiang TJ and Zheng S: The optimal combination of serum tumor markers with bioinformatics in diagnosis of colorectal carcinoma. J Zhejiang Univ 33: 407-410, 2004 (In Chinese)

17. Newton KF, Newman W and Hill J: Review of biomarkers in colorectal cancer. Colorectal Dis 14: 3-17, 2012.

18. Wiggers T, Arends JW, Verstijnen C, et al: Prognostic significance of CEA immunoreactivity patterns in large bowel carcinoma tissue. Br J Cancer 54: 409-414, 1986.

19. Kitadai Y, Radinsky R, Bucana CD, et al: Regulation of carcinoembryonic antigen expression in human colon carcinoma cells by the organ microenvironment. Am J Pathol 149: 1157-1166, 1996.

20. Seregni E, Ferrari L, Martinetti A and Bombardieri E: Diagnostic and prognostic tumor markers in the gastrointestinal tract. Semin Surg Oncol 20: 147-166, 2001.

21. Araki K, Furuya Y, Kobayashi M, et al: Comparison of mucosal microvasculature between the proximal and distal human colon. J Electron Microsc (Tokyo) 45: 202-206, 1996.

22. Skinner SA and O'Brien PE: The microvascular structure of the normal colon in rats and humans. J Surg Res 61: 482-490, 1996.

23. Devesa SS and Chow WH: Variation in colorectal cancer incidence in the United States by subsite of origin. Cancer 71: 3819-3826, 1993

24. Shamsuddin AM, Phelps PC and Trump BF: Human large intestinal epithelium: light microscopy, histochemistry, and ultrastructure. Hum Pathol 13: 790-803, 1982.

25. Macfarlane GT, Gibson GR and Cummings JH: Comparison of fermentation reactions in different regions of the human colon. J Appl Bacteriol 72: 57-64, 1992.

26. Mercurio MG, Shiff SJ, Galbraith RA and Sassa S: Expression of cytochrome P450 mRNAs in the colon and the rectum in normal human subjects. Biochem Biophys Res Commun 210: 350-355, 1995.

27. Cancer Genome Atlas Network: Comprehensive molecular characterization of human colon and rectal cancer. Nature 487: 330-337, 2012. 